Appeared in: Computers and Geotechnics, 2012, 42: 44-51.

\title{
Reliability updating in geotechnical engineering including spatial variability of soil
}

\author{
Iason Papaioannou ${ }^{1,2^{*}}$, Daniel Straub ${ }^{1}$
}

1 Engineering Risk Analysis Group, Technische Universität München, 80290 Munich, Germany

${ }^{2}$ Chair of Computation in Engineering, Technische Universität München, 80290 Munich, Germany

*Corresponding author. Email: iason.papaioannou@tum.de

\begin{abstract}
At geotechnical sites, deformation measurements are routinely performed during the construction process. In this paper, it is shown how information from such measurements can be utilized to update the reliability estimate of the geotechnical site at future construction stages. A recently proposed method for Bayesian updating of the reliability is successfully applied in conjunction with a stochastic nonlinear geotechnical finite element model. Therein, uncertainty in the soil material properties is modelled by non-Gaussian random fields. The structural reliability evaluations required for the Bayesian updating are carried out by means of subset simulation, an efficient adaptive Monte Carlo method. The approach is demonstrated through an application to a sheet pile wall at a deformation-sensitive geotechnical construction site.
\end{abstract}

\section{Keywords}

Reliability updating, equality information, finite elements, random fields, subset simulation. 


\section{Introduction}

Geotechnical engineers commonly use the observation method to deal with uncertainties in site conditions or performance behavior. The engineer collects a few hypotheses about site conditions and then gathers field observations (e.g. measurements of deformations, stresses or other relevant data) to identify the correct hypothesis. In probability theory, Bayesian updating formalizes this procedure, by quantifying the effect of an observation on the probability of a prior hypothesis. Bayesian updating is applied here within the structural reliability concept. The goal is the computation of the reliability of the geotechnical site conditional on the available information obtained in-situ. To this end, a recently proposed methodology [1] that enables updating of the reliability given measurement information is applied. Reliability updating in geotechnical engineering was considered previously [2-4], but was either limited to information of the inequality type (i.e. knowledge on the exceedance of a failure condition [2] or on historically survived load conditions [3]), or to a small number of measurements [4]. The method presented in [4] is applicable in real-time, but becomes inefficient in the case where measurements deviate significantly from the expected value. In contrast, the applicability of the methodology used in this paper for reliability updating with measurements does not depend on the amount or type of information.

In geotechnical analysis, the largest uncertainties usually stem from the mechanical properties of the soil materials. In addition, the uncertain material properties tend to vary in space, even within homogeneous layers. Several studies have shown that the spatial variability of the soil has an important influence on the computed reliability [5-7]. An accurate representation of the spatial variability of the uncertain soil material properties requires a random field modelling. If a stochastic discretization of the random fields is employed as part of a finite element (FE) reliability analysis procedure, a large number of 
random variables may be required. An efficient method for dealing with such highdimensional problems is the subset simulation [8].

In this work, subset simulation is applied to the reliability updating of a nonlinear geotechnical FE model of an excavation in sand with a sheet pile retaining wall. Thereby, the reliability of the geotechnical construction in its critical stage is computed conditional on deformation measurements made at an intermediate excavation depth.

\section{Stochastic FE modelling}

\subsection{Mechanical modelling}

In computational mechanics, the continuous nature is approximated by a discrete representation through the discretization procedure. The FE method [9] is a widely used discretization method in geotechnical engineering applications. It proceeds by splitting the continuous medium into an assembly of individual elements, as defined by the FE mesh. Nonlinear FE analysis computes the deformations and stresses through the solution of a nonlinear system of equations, wherein the nonlinear effects of the soil material properties as well as the interaction between the soil and adjacent retaining structures are taken into account. For the simulation of geotechnical sites, usually a sequence of nonlinear FE analyses is performed to model the different stages of the construction process.

In this study, the deterministic mechanical modelling is carried out in $2 \mathrm{D}$ using the SOFiSTiK software [10]. The soil is modelled with elasto-plastic plain strain finite elements and its shear strength is considered to be governed by the Mohr-Coulomb criterion. Retaining structures are represented by beam finite elements, while their interface with the adjacent soil is modelled by interface elements featuring a reduced shear capacity compared to the soil base material. The FE analysis mimics the staged construction process. The in-situ stress state is modelled first; then the retaining structure is installed by activating the corresponding beam 
and interface elements. Finally, the elements corresponding to the excavation trench are removed in a stepwise procedure, where in each step the necessary loading is applied to establish equilibrium. A more detailed description of the applied deterministic FE model is given in Section 5.

\subsection{Random fields}

The probabilistic description of the mechanical properties of the soil material requires the consideration of random fields. A random field $X(\mathbf{t})$ is defined as a collection of random variables indexed by a continuous parameter $\mathbf{t} \in \Omega \subset \mathbb{R}^{d}(d=2$ or 3$)$, i.e. $\mathbf{t}$ is a location vector. To completely define a random field, the joint distribution of the random variables $\left\{X\left(\mathbf{t}_{1}\right), X\left(\mathbf{t}_{2}\right) \ldots, X\left(\mathbf{t}_{n}\right)\right\}$ for any $\left\{n, \mathbf{t}_{1}, \mathbf{t}_{2}, \ldots, \mathbf{t}_{n}\right\}$ must be specified. This is straightforward when the field is Gaussian (i.e. when the random variables $\left\{X\left(\mathbf{t}_{1}\right), X\left(\mathbf{t}_{2}\right) \ldots, X\left(\mathbf{t}_{n}\right)\right\}$ have the multivariate normal distribution) and the mean and covariance functions are available. In the case where the marginal distribution of $X(\mathbf{t})$ is not Gaussian, the specification requires an assumption on the form of the joint distribution. A class of random fields with given nonGaussian marginal distribution and covariance function can be defined as a nonlinear transformation of an underlying Gaussian field [11] of the form:

$$
X(\mathbf{t})=F^{-1}\{\Phi[Z(\mathbf{t})]\}
$$

where $F^{-1}($.$) is the inverse of the given marginal distribution, \Phi($.$) is the standard normal$ distribution function and $Z(\mathbf{t})$ is an underlying Gaussian field with zero mean and unit variance. The joint distribution defined by this transformation is called the Nataf multivariate distribution $[12,13]$ or alternatively is said to be of the Gaussian copula class. Note that the specification of the Nataf distribution involves the estimation of the correlation structure of the field $Z(\mathbf{t})$, by solving an integral equation. 
A random field is said to be weakly homogeneous if its probabilistic structure is invariant to a shift of the parameter origin up to a second order. A direct consequence of this is that the mean and variance of the field are constant over space and the spatial variability can be defined by means of the auto-correlation coefficient function $\rho_{X X}(\tau)$, where $\boldsymbol{\tau}\left(=\left[\tau_{x}, \tau_{y}, \tau_{z}\right]^{T}\right.$ for $d=3$ ) is the vector containing the location differences in each direction [14].

\subsection{Discretization of random fields}

The continuous random field $X(\mathbf{t})$ may be approximated by a discrete $\hat{X}(\mathbf{t})$, defined in terms of a finite set of random variables $\left\{X_{1}, X_{2}, \ldots, X_{n}\right\}$. Several methods have been proposed for the discretization of random fields - a comprehensive review is given in [15]. In this study, the midpoint method [16] is adopted. Like in the deterministic FE method, this stochastic discretization method requires the splitting of the domain into a discrete assembly of elements, which is usually referred to as the stochastic finite element (SFE) mesh. It should be noted that the SFE mesh can be independent of the FE mesh used for performing the mechanical analysis for a realization of the random field - to avoid confusion we will call this latter mesh deterministic FE mesh. In the midpoint method, the value of the random field $X(\mathbf{t})$ over each SFE $i$ is represented by its value at the midpoint (centre of gravity) $\mathbf{t}_{i}$ of the element:

$$
X_{i}=X\left(\mathbf{t}_{i}\right)
$$

If the random field is weakly homogeneous, the mean and the variance of the random variables $\left\{X_{1}, X_{2}, \ldots, X_{n}\right\}$ are constant over the entire field and thus not affected by the discretization. The entries $C_{i j}$ of the correlation matrix $\mathbf{C}$ of the random variables are evaluated as follows:

$$
C_{i j}=\rho_{X X}\left(\boldsymbol{\tau}_{i j}\right)
$$


where $\boldsymbol{\tau}_{i j}=\mathbf{t}_{i}-\mathbf{t}_{j}$ and $\left\{\mathbf{t}_{i}, \mathbf{t}_{j}\right\}$ are the midpoints of elements $i$ and $j$ respectively. When the marginal distribution $F(x, \mathbf{t})$ of the random field is given, the marginal distribution of each random variable $X_{i}$ will be $F\left(x, \mathbf{t}_{i}\right)$. Moreover, if the random field is homogeneous, then the marginal distribution will be constant over the entire field. The random field is thus reduced to a vector $\mathbf{X}=\left[X_{1}, X_{2}, \ldots, X_{n}\right]^{T}$ of correlated random variables with known marginal distributions and correlation matrix $\mathbf{C}$.

The midpoint method is shown to over-represent the variance of any response quantity [16]. This is due to the fact that a full correlation between different points within each SFE is implied. In addition, each realization of the approximated field is piecewise constant, leading to discontinuities arising at the boundaries of each element. However, the fact that the field remains constant over each SFE facilitates the definition of the random field within the context of a "black-box" FE solver, since all finite elements within each SFE will have the same random material properties. This can also be achieved by the spatial average method [14], which describes the field within each SFE in terms of the spatial average of the field over the SFE. However, in the case of non-Gaussian random fields, the marginal distribution of the spatial average of the field is almost impossible to obtain [14]. In this case, a transformation of the form of Eq. (1) to an equivalent Gaussian field must be performed prior to the discretization. This is not necessary when the midpoint method is employed, since the marginal distribution is not affected by the discretization; instead it is sufficient to apply the transformation to the discrete random vector $\mathbf{X}$ describing the random field $X(\mathbf{t})$.

The selection of the SFE mesh is based on the expected rate of fluctuation, which in the case of homogeneous fields is described by the auto-correlation coefficient function (guidelines for several discretization methods and common correlation models are given in [12]). However, the SFE mesh should be coarse enough to avoid near perfect correlation between the elements, which may cause instability in the probabilistic transformation. Obviously, these requirements differ considerably from the ones for the selection of the 
deterministic FE mesh. In this study, the SFE mesh is chosen first and each SFE is defined in the SOFiSTiK program as a structural area with random material properties. Then, the structural areas are meshed by the SOFiSTiK program, resulting in the deterministic FE mesh.

Fig.1 shows realizations of a weakly homogeneous random field representing the Young's modulus $E$ of a soil, whereby the stochastic discretization is performed by application of the midpoint method. This random field is anisotropic, because the correlation length in the horizontal direction is larger than in the vertical direction. The details of this random field are given in Section 5.1.
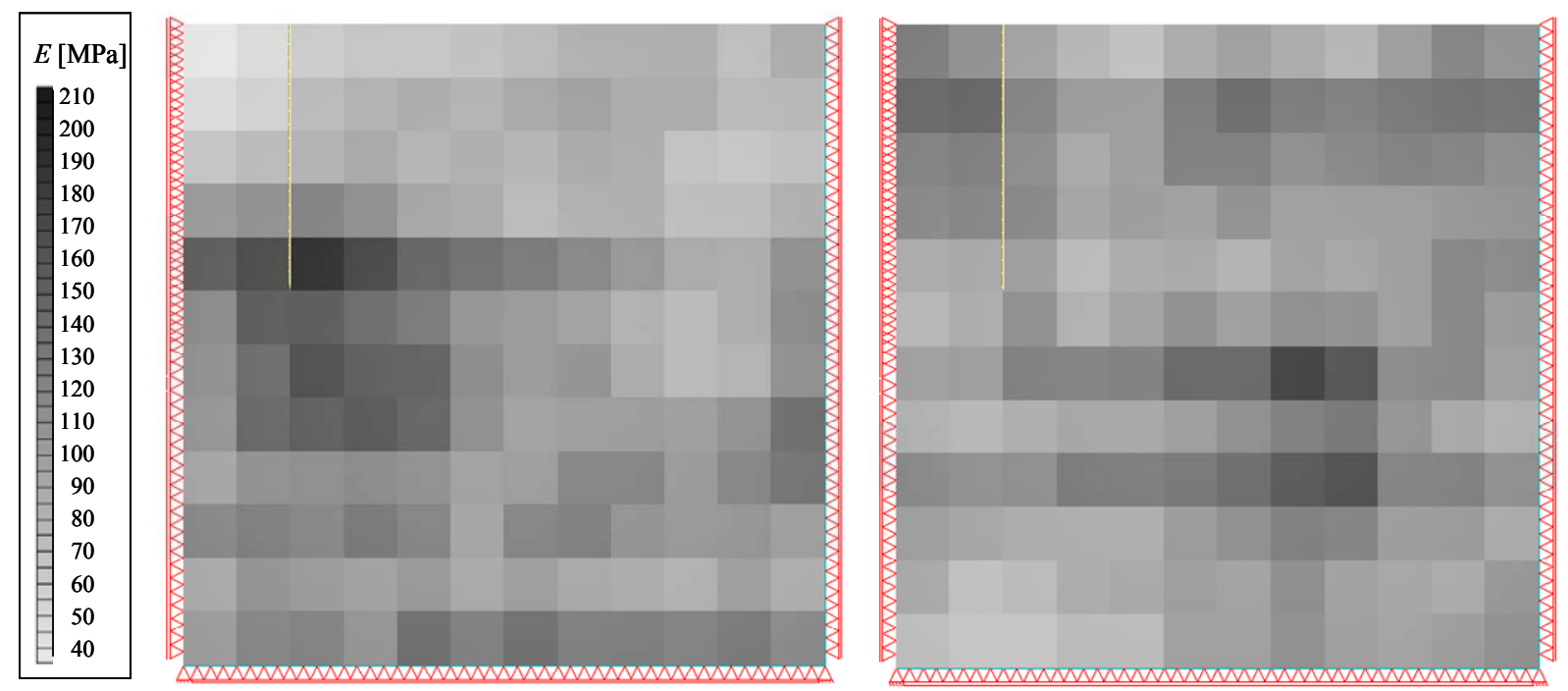

Fig. 1. Two realizations of a homogeneous anisotropic lognormal random field representing the Young's modulus $E$ of a soil. 


\section{Reliability Analysis}

Let $\mathbf{X}$ be the vector of random variables of a geotechnical model, with joint probability density function (PDF) $f_{\mathbf{X}}(\mathbf{x})$. A failure event $F$ is defined by a domain $\Omega_{F}=\{g(\mathbf{x}) \leq 0\}$ in the outcome space of $\mathbf{X}$, with $g(\mathbf{x})$ being a limit-state function, representing the failure condition of the system. In the case where only one limit-state function is considered, the problem is termed component reliability problem. If the domain $\Omega_{F}$ is given by the intersection of $m$ domains with corresponding limit-state functions $\left\{g_{i}(\mathbf{x}), i=1, \ldots, m\right\}$, defining a parallelsystem reliability problem, the failure event can be specified by the following equivalent limit-state function:

$$
g(\mathbf{x})=\max _{1 \leq i \leq m} g_{i}(\mathbf{x})
$$

Similar formulations are available for general system reliability problems and can be found in the literature [17]. The methods presented in this paper are directly applicable to any of these system formulations.

Once the failure domain is defined, the probability of failure can be evaluated by:

$$
\operatorname{Pr}(F)=\operatorname{Pr}\{g(\mathbf{X}) \leq 0\}=\int_{\Omega_{F}} f_{\mathbf{X}}(\mathbf{x}) \mathrm{d} \mathbf{x}
$$

If the joint distribution of the random variables $\mathbf{X}$ is described by the Nataf model, a transformation of the random variables $\mathbf{X}$ from the original random variable space to an equivalent independent standard Gaussian space $\mathbf{U}=\mathbf{T}(\mathbf{X})$ is straightforward [13]. In the present case, where the random field is reduced to a random vector applying the midpoint method, this mapping involves a transformation equivalent to the one of Eq. (1). Each realization $\mathbf{u}$ of the independent standard normal random variables $\mathbf{U}$ can be transformed to a realization in the original random variable space as $\mathbf{x}=\mathbf{T}^{-1}(\mathbf{u})$. The failure event can then be 
described by a domain in the transformed space of $\mathbf{U}$ as $\Omega_{F}=\left\{g\left[\mathbf{T}^{-1}(\mathbf{u})\right] \leq 0\right\}$ and the probability may be computed as follows:

$$
\operatorname{Pr}(F)=\int_{G(\mathbf{u}) \leq 0} \varphi_{\mathbf{U}}(\mathbf{u}) \mathrm{d} \mathbf{u}
$$

where $\varphi_{\mathbf{U}}(\mathbf{u})$ is the standard normal joint PDF and $G(\mathbf{u})=g\left[\mathbf{T}^{-1}(\mathbf{u})\right]$ is the limit state function in the $\mathbf{U}$-space. Structural reliability methods have been developed, which enable an efficient evaluation of the integrals in Eq. (5) and (6) even for high dimensions of $\mathbf{X}$ and $\mathbf{U}$. The standard reliability measure used in structural reliability is the generalized reliability index, defined as the following decreasing function of $\operatorname{Pr}(F)$ [17]:

$$
\beta=-\Phi^{-1}[\operatorname{Pr}(F)]
$$

where $\Phi^{-1}($.$) is the inverse of the standard normal cumulative distribution function.$

\subsection{Reliability updating with equality information}

Measurements and other observations of the structural or geotechnical system can be considered an event $Z$. In the context of structural reliability, this event $Z$ is defined through a domain $\Omega_{Z}$ in the outcome space of the basic random variables $\mathbf{X}$, in analogy to the definition of the failure event. The conditional probability of $F$ given the information $Z$ is:

$$
\operatorname{Pr}(F \mid Z)=\frac{\operatorname{Pr}(F \cap Z)}{\operatorname{Pr}(Z)}=\frac{\int_{\Omega_{F}} f_{\Omega_{Z}}(\mathbf{x}) \mathrm{d} \mathbf{x}}{\int_{\Omega_{Z}} f_{\mathbf{X}}(\mathbf{x}) \mathrm{d} \mathbf{x}}
$$

The domain $\Omega_{Z}$ is defined through a limit state function $h(\mathbf{x})$; if $\Omega_{Z}$ is defined as $\Omega_{Z}=\{h(\mathbf{x}) \leq 0\}$, the information event $Z$ is said to be of the inequality-type and if $\Omega_{Z}$ is defined as $\Omega_{Z}=\{h(\mathbf{x})=0\}, Z$ is said to be of the equality-type. Quantitative measurements are 
commonly of the equality-type. For equality-type information, the probability of the event $Z$ is zero and both integrals in Eq. (8) will result in zero. Direct application of structural reliability methods is thus not possible. To circumvent this problem, the method proposed in [1] will be applied, according to which the event $Z$ is replaced by an equivalent inequality-type event $Z_{e}$ with non-zero probability. The method is exact, i.e. it can be shown that $\operatorname{Pr}(F \mid Z)=\operatorname{Pr}\left(F \mid Z_{e}\right)$.

To illustrate the methodology, consider the case where $Z$ describes the measurement $s_{m}$ of a system characteristic $s(\mathbf{x})$, e.g. the deformation of a retaining wall. Assuming that this measurement has an additive random measurement error $\varepsilon_{m}$, the corresponding equality-type limit state function is:

$$
h\left(\mathbf{x}, \varepsilon_{m}\right)=s(\mathbf{x})-s_{m}+\varepsilon_{m}
$$

This is the expression commonly used in structural reliability. Alternatively, the information of the event $Z$ with respect to the random variables $\mathbf{X}$ can be expressed by the likelihood function:

$$
L(\mathbf{x})=f_{\varepsilon_{m}}\left[s_{m}-s(\mathbf{x})\right]
$$

where $f_{\varepsilon m}$ is the PDF of $\varepsilon_{m}$. It is proposed in [1] to utilize this likelihood function to define a new limit state function:

$$
h_{e}(\mathbf{x}, u)=u_{a}-\Phi^{-1}[c L(\mathbf{x})]
$$

where $u_{a}$ is the outcome of a standard normal random variable $U_{a}$ and $c$ is a positive constant, chosen to ensure that $c L(\mathbf{x}) \leq 1$ for all $\mathbf{x}$; with the likelihood function of Eq. (10) it must hold $0<c \leq\left[\max f_{\varepsilon m}\left(\varepsilon_{m}\right)\right]^{-1}$. Let $\Omega_{Z e}=\left\{h_{e}\left(\mathbf{x}_{+}\right) \leq 0\right\}$, be the corresponding domain defining the event $Z_{e}$, where $\mathbf{x}_{+}$is the outcome of $\mathbf{X}_{+}=\left[\mathbf{X}, U_{a}\right]^{T}$. This event is equivalent to the original event $\mathrm{Z}$ when applied for Bayesian updating. Specifically, it is shown in [1] that the conditional probability of $F$ given $Z$ is: 


$$
\operatorname{Pr}(F \mid Z)=\operatorname{Pr}\left(F \mid Z_{e}\right)=\frac{\operatorname{Pr}\left(F \cap Z_{e}\right)}{\operatorname{Pr}\left(Z_{e}\right)}=\frac{\int f_{F} \cap \Omega_{\mathbf{X}_{+}}\left(\mathbf{x}_{+}\right) \mathrm{d} \mathbf{x}_{+}}{\int_{\Omega_{Z e}} f_{\mathbf{X}_{+}}\left(\mathbf{x}_{+}\right) \mathrm{d} \mathbf{x}_{+}}
$$

where $f_{\mathbf{X}+}\left(\mathbf{x}_{+}\right)$is the joint PDF of $\mathbf{X}_{+}$. As opposed to the original formulation in Eq. (8), both integrals in Eq. (12) have non-zero values and can thus be computed using any structural reliability method, including simulation methods. Note that the approach can be directly extended to cases where several equality-type observations are available, by defining the equivalent inequality-type limit-state functions for all observations [1].

For practical implementation, the two integrals in Eq. (12) are computed in the space of standard normal random variables $\mathbf{U}$, in analogy to the computation of the failure probability in Eq. (6). To this end, the limit state function $h_{e}\left(\mathbf{x}_{+}\right)$is transformed to the standard normal space by $H_{e}\left(\mathbf{u}_{+}\right)=h_{e}\left(\mathbf{T}^{-1}(\mathbf{u}), u_{a}\right)$. The conditional probability of failure then becomes:

$$
\operatorname{Pr}(F \mid Z)=\frac{\int_{\max \left[G\left(\mathbf{u}_{+}\right), H_{e}\left(\mathbf{u}_{+}\right)\right] \leq 0} \varphi_{\mathbf{u}_{+}}\left(\mathbf{u}_{+}\right) \mathrm{d} \mathbf{u}_{+}}{\int_{H_{e}\left(\mathbf{u}_{+}\right) \leq 0} \varphi_{\mathbf{u}_{+}}\left(\mathbf{u}_{+}\right) \mathrm{d} \mathbf{u}_{+}}
$$

wherein the parallel system formulation of Eq. (4) is used to represent the intersection in the nominator. Here, subset simulation is applied to solve the two integrals in Eq. (13).

\section{Reliability analysis in high dimensions}

The stochastic discretization of a random field can lead to a large number of random variables, depending on its auto-correlation coefficient function. The reliability of nonlinear problems involving a large number of random variables can be solved efficiently by either first- or second-order reliability methods combined with the direct differentiation method for the evaluation of the derivatives (see [18], [19]) or by selected simulation approaches, 
including subset simulation [8], spherical subset simulation [20] and recently the asymptotic sampling method [21]. First/second order reliability methods using direct differentiation require alterations at the FE code level, while simulation approaches may be easily coupled with a "black-box" FE code. In this paper, the subset simulation is employed for the estimation of the probabilities in Eq. (13).

\subsection{Subset simulation}

The subset simulation is an adaptive simulation method developed by Au and Beck [8], which is shown to be efficient especially in high dimensions. The method is based on the standard Monte Carlo simulation (MCS) but overcomes its inefficiency in estimating small probabilities, while maintaining its independency on the problem dimensionality. This is achieved by expressing the failure event $F$ as the intersection of $M$ intermediate failure events:

$$
F=\bigcap_{i=1}^{M} F_{i}
$$

where $F_{1} \supset F_{2} \supset \cdots \supset F_{M}=F$. The probability of failure is estimated by computing the joint probability $\operatorname{Pr}\left(\bigcap_{i=1}^{M} F_{i}\right)$ as a product of conditional probabilities:

$$
\operatorname{Pr}(F)=\operatorname{Pr}\left(\bigcap_{i=1}^{M} F_{i}\right)=\operatorname{Pr}\left(F_{1}\right) \prod_{i=2}^{M} \operatorname{Pr}\left(F_{i} \mid F_{i-1}\right)
$$

The failure event is defined in the equivalent standard normal space by $G(\mathbf{u}) \leq 0$; each of the intermediate events is defined as $F_{i}=\left\{G(\mathbf{u}) \leq c_{i}\right\}$, where $c_{1}>\ldots>c_{M}=0$. The values of $c_{i}$ are chosen adaptively so that the estimates of the conditional probabilities correspond to a chosen value $p_{0}$. The probability of $F_{1}$ is computed by applying the crude MCS method. Through MCS, $N$ samples of $\mathbf{U}$ are simulated and $c_{1}$ is set equal to the $\left[\left(1-p_{0}\right) N\right]$-th largest value among the samples $\left\{G\left(\mathbf{u}_{k}\right): k=1, \ldots, N\right\}$. The $p_{0} N$ samples $\mathbf{u}_{i}$ for which $G\left(\mathbf{u}_{i}\right) \leq c_{1}$ are used as starting points for the simulation of $\left(1-p_{0}\right) N$ samples conditional on $F_{1}$, by applying the 
Metropolis-Hastings algorithm ([22],[23]), which is a Markov Chain Monte Carlo (MCMC) technique. This procedure is repeated for sampling conditional on $F_{2}, F_{3}, \ldots$ until the maximum level $M$ is reached, for which the threshold $c_{M}=0$ is given. The failure probability is then approximated by:

$$
\operatorname{Pr}(F) \approx p_{0}{ }^{M-1} \operatorname{Pr}\left(F_{M} \mid F_{M-1}\right)
$$

where the conditional probability $\operatorname{Pr}\left(F_{M} \mid F_{M-1}\right)$ is equal to the ratio of the number of samples for which $G(\mathbf{u}) \leq 0$ over the total number of samples $N$ simulated conditional on $F_{M-1}$.

The analyst is free in the choice of $p_{0}$ and the number of samples at each step $N$. However, $N$ should be selected large enough to give an accurate estimate of $p_{0}$. In this study, the values $p_{0}=0.1$ and $N=500$ are chosen, following the initial suggestions given in [8]. Therefore, if the magnitude of $\operatorname{Pr}(F)$ is in the order of $10^{-k}$ then the total required number of deterministic FE analyses $N_{\text {tot }}$ is $N_{\text {tot }}=k\left(1-p_{0}\right) N+N=450 k+500$. Note that for the crude MCS, the required number of samples for the same failure probability would be $N_{t o t}=10^{k+2}$, which indicates that the gain in efficiency of the subset simulation can be of several orders of magnitude.

\section{Application}

The illustrative application is an excavation in sand with a sheet pile retaining wall, where deformation measurements made at an intermediate excavation depth are utilized to update the reliability of the construction site at the stage of full excavation. The corresponding FE model is implemented in the SOFiSTiK program and the reliability assessment is performed using a reliability tool, developed as part of the SOFiSTiK software package [24]. 


\subsection{Geotechnical model description}

The site consists of a $5.0 \mathrm{~m}$ deep trench with cantilever sheet piles, without anchors or bottom support (Fig. 2), in a homogeneous soil layer of dense cohesionless sand with uncertain spatially varying mechanical properties. The soil is modelled in $2 \mathrm{D}$ with plane-strain finite elements. For simplicity, neither groundwater nor external loading is considered. Additionally, we take advantage of the symmetry of the trench and model just one half of the soil profile. However, it should be noted that this is an approximation when randomness in the soil material is taken into account. The material model used is an elasto-plastic model with a prismatic yield surface according to the Mohr-Coulomb criterion and a non-associated plastic flow. The probabilistic models of the material properties of the soil are shown in Table 1 . The spatial variability of the soil is modelled by homogeneous random fields, with the following exponential auto-correlation coefficient function [14]:

$$
\rho_{X X}(\tau)=\exp \left(-\frac{\tau_{x}}{l_{x}}-\frac{\tau_{z}}{l_{z}}\right)
$$

where $\tau=\left[\tau_{x}, \tau_{z}\right]^{T}$ is the vector of absolute distances in the $x$ (horizontal) and $z$ (vertical) directions. The correlation lengths are $l_{x}=20 \mathrm{~m}$ and $l_{z}=5 \mathrm{~m}$ for all uncertain soil material properties: specific weight $\gamma$, Young's modulus $E$ and friction angle $\varphi$. An infinite correlation length is intrinsically assumed in the $y$ direction (out of plane). Cross-correlation between the different material properties is not included. The joint distribution of the random variables in the random fields is the Nataf distribution, with marginal distributions according to Table 1 . The random fields are discretized by the midpoint method using a SFE mesh, consisting of 144 deterministic FE patches. The stochastic discretization resulted in a total of $3 \times 144=432$ basic random variables. In Fig. 3, the stochastic and deterministic FE meshes are shown. Fig. 4 shows plots of the auto-correlation coefficient function and its corresponding 
approximation. In Fig. 1, two realizations of the lognormal random field representing the uncertainty of the Young's modulus are shown.

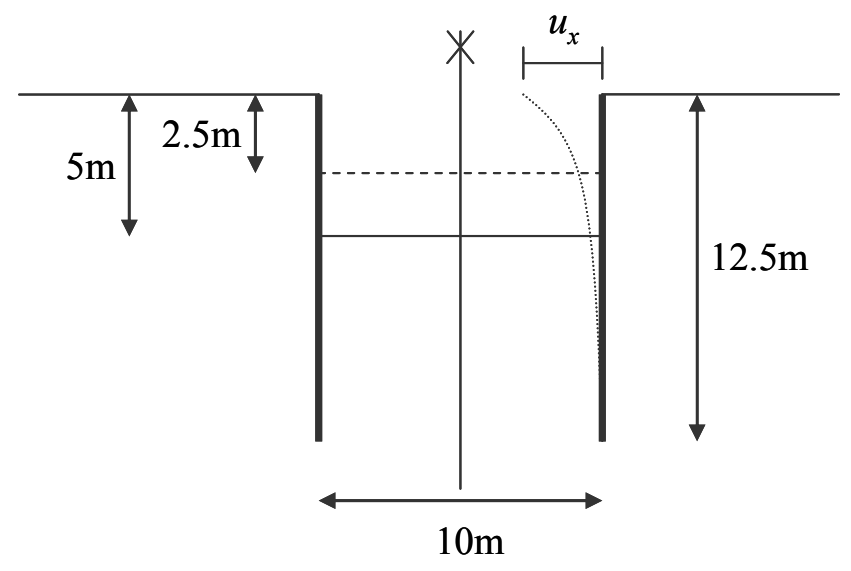

Dense cohesionless sand

Fig. 2. Sheet pile wall in sand.

Table 1. Material properties.

\begin{tabular}{llll}
\hline Parameter & Distribution & Mean & COV \\
\hline Specific weight $\gamma\left[\mathrm{kN} / \mathrm{m}^{3}\right]$ & Normal & 19.0 & $5 \%$ \\
Young's modulus $E[\mathrm{MPa}]$ & Lognormal & 125.0 & $25 \%$ \\
Poisson's ratio $v$ & - & 0.35 & - \\
Friction angle $\varphi\left[^{\circ}\right]$ & Beta $(0.0,45.0)$ & 35.0 & $10 \%$ \\
Cohesion $c[\mathrm{MPa}]$ & - & 0.0 & - \\
Dilatancy angle $\psi\left[^{\circ}\right]$ & - & 5.0 & - \\
\hline
\end{tabular}


The sheet pile dimension and profile is determined analytically using the conventional method for cantilever sheet pile design in granular soils, which requires equilibrium of the active and passive lateral pressures [25]. Applying a global safety factor of 1.5, the design results in sheet piles of depth of $7.5 \mathrm{~m}$ and profile PZC 13. The Young's modulus of steel is taken as $210 \mathrm{GPa}$. The pile is modelled using beam elements with an equivalent rectangular cross section that behaves equally to the sheet pile in bending and axial resistance. The interaction between the retaining structure and the surrounding soil is modelled using nonlinear interface elements. An elastoplastic model with a yield surface defined by the Mohr-Coulomb criterion is used to describe the interface behavior. The elastic properties of the interface elements are taken from the mean values of the adjacent soil, while the strength properties are reduced by the factor $2 / 3$ and a zero dilatancy is chosen.

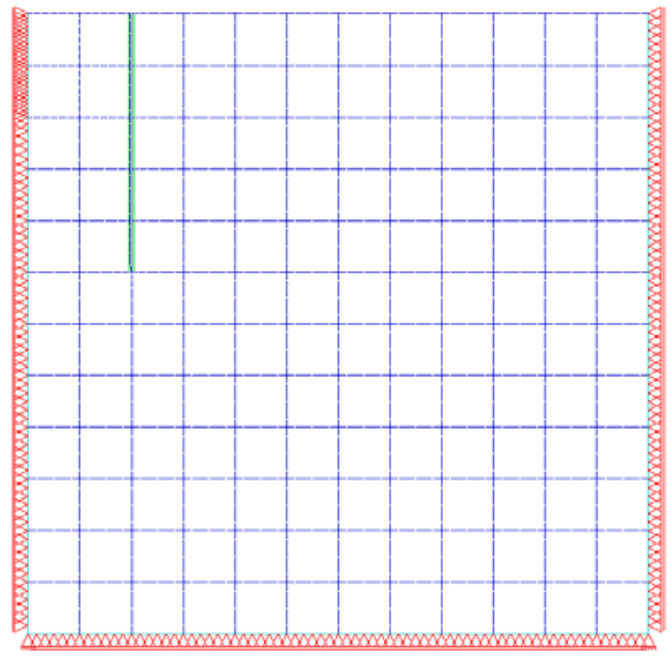

(a) Stochastic finite element mesh

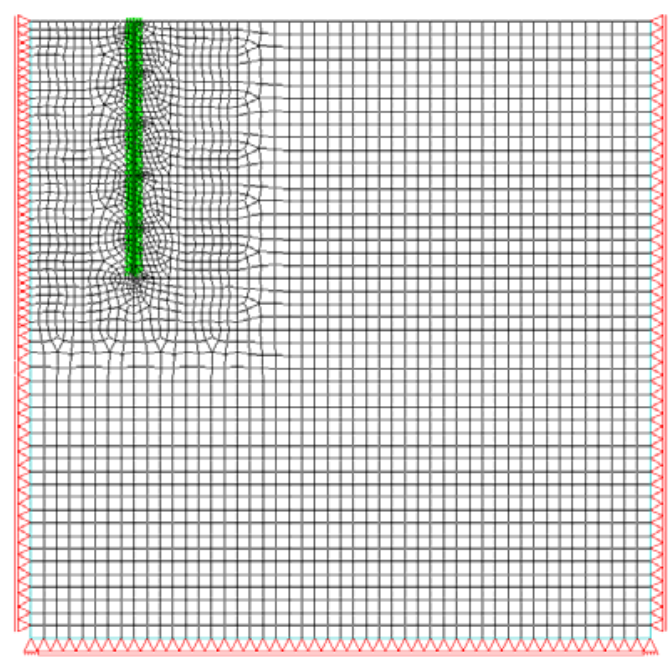

(b) Deterministic finite element mesh

Fig. 3. Stochastic and deterministic finite element mesh. 


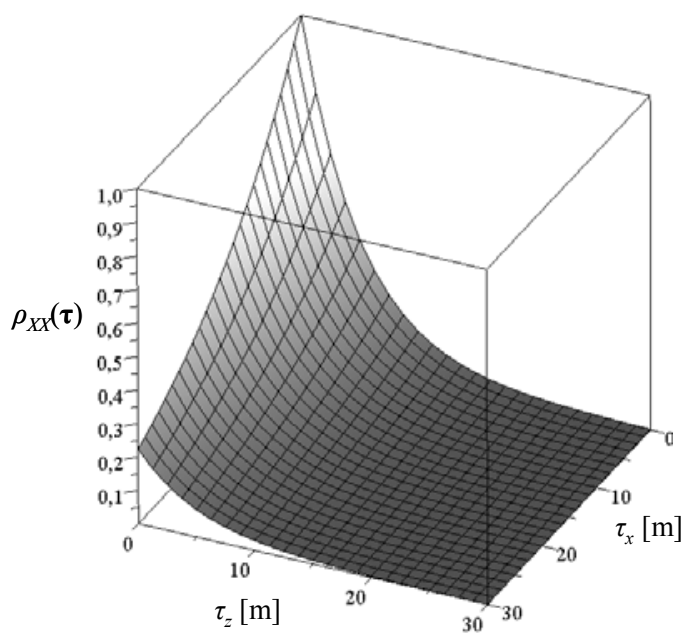

(a) Original correlation structure

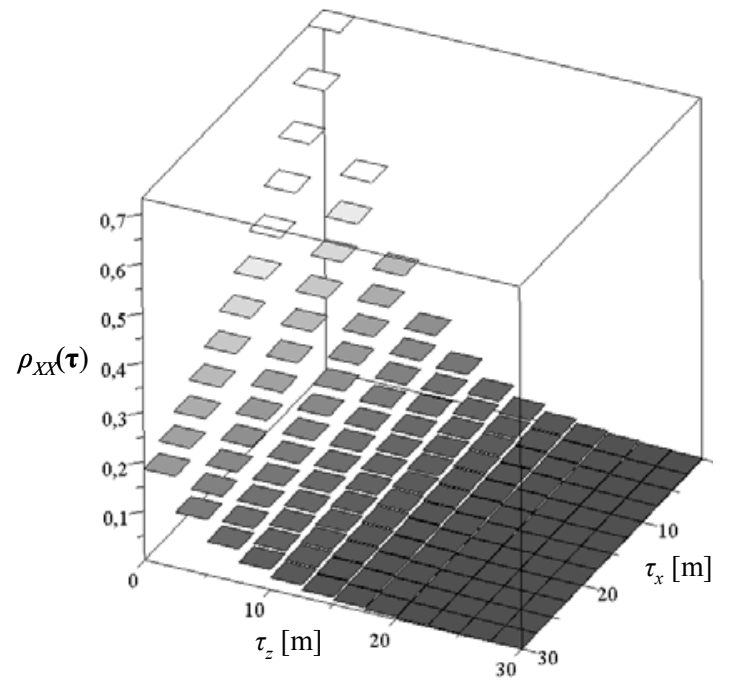

(b) Midpoint method approximation

Fig. 4. Approximation of the correlation structure by the midpoint method.

The finite element analysis is performed stepwise, following the construction process. First, the modelling of the in-situ stress state is carried out by means of the $K_{0}$-procedure, where $K_{0}$ is the lateral earth pressure coefficient at rest, computed here using the expression proposed by Jaky [26] for normally consolidated soils:

$$
K_{0}=1-\sin \varphi
$$

Next, the sheet pile is installed by activating the corresponding beam and interface elements. Finally, the excavation is modelled by removing the plane-strain elements corresponding to the trench and applying the necessary loading to establish equilibrium. 


\subsection{Limit-state functions}

The maximum horizontal $u_{x}$ displacement occurs at the top of the trench. The failure event $F$ is defined as the event of $u_{x}$ exceeding a threshold of $u_{x, t}=0.1 \mathrm{~m}$. Mathematically, this is expressed through the following limit state function:

$$
g(\mathbf{x})=u_{x, t}-u_{x}(\mathbf{x})
$$

This is a serviceability limit state, reflecting the assumed serviceability design requirements. A stability analysis, performed by application of the shear strength reduction technique [27] with the mean values of the random fields, resulted in a factor of safety of 2.5. In Eq. (19), $u_{x}(\mathbf{x})$ is evaluated by the $\mathrm{FE}$ analysis for given values of the random variables $\mathbf{X}$. We assume that a measurement of the displacement $u_{x}$ is made at an intermediate excavation step of $2.5 \mathrm{~m}$ depth. This information is expressed by an event $Z$, described by the following likelihood function:

$$
L(x)=\varphi\left\{\left[u_{x, m}-u_{x}(\mathbf{x})\right] / \sigma_{\varepsilon, m}\right\} / \sigma_{\varepsilon, m}
$$

where $\sigma_{\varepsilon, m}$ is the standard deviation of the measurement error, which is a zero mean Gaussian random variable; $\varphi($.$) is the standard normal PDF. The corresponding equivalent inequality$ limit state function is obtained according to Eq. (11):

$$
h_{e}(\mathbf{x}, u)=u_{a}-\Phi^{-1}\left[\frac{c}{\sigma_{\varepsilon, m}} \varphi\left(\frac{u_{x, m}-u_{x}(\mathbf{x})}{\sigma_{\varepsilon, m}}\right)\right]
$$

where $u_{a}$ is the realization of the auxiliary standard normal random variable. The constant is chosen as $c=\sigma_{\varepsilon, m}$, which satisfies the condition $c L(\mathbf{x}) \leq 1$. 


\subsection{Results and discussion}

Figure 5 depicts the deformed configuration at the final excavation stage computed with the mean values of the random fields. This analysis gives a first-order approximation of the mean (expected) displacements. At the top of the trench, the estimated mean value of the horizontal displacement $u_{x}$ is $50.2 \mathrm{~mm}$.

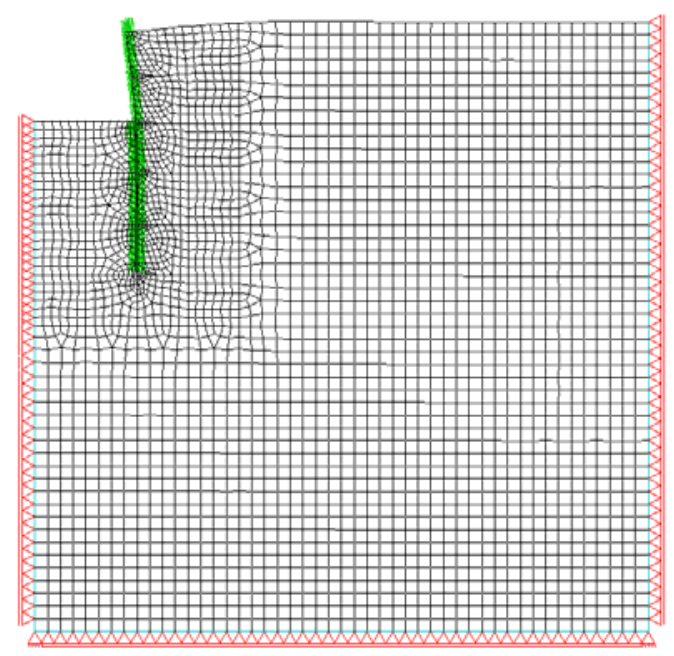

Fig. 5. Magnified deformed configuration at the final excavation step.

The reliability analysis is performed by means of subset simulation. Without measurements, the computed failure probability is $\operatorname{Pr}(F)=1.36 \times 10^{-2}$ with a corresponding reliability index $\beta=2.21$

For the estimation of the updated failure probability conditional on the measurement event $Z$, the integrals in Eq. (13) were evaluated with subset simulation. The reliability updating was performed for different measurement outcomes $u_{x, m}$, and different values of the standard deviation $\sigma_{\varepsilon, m}$ of the measurement error. The results are summarized in Table 2 and the computed reliability indices are plotted in Fig. 6. For comparison, the (a-priori) first-order approximation of the expected value of the measurement outcome $u_{x, m}$ is computed as $2.6 \mathrm{~mm}$. 
Table 2. Updated failure probability $\left[\operatorname{Pr}(F)=1.360 \times 10^{-2}\right]$.

\begin{tabular}{lllll}
\hline Measurement & $\sigma_{\varepsilon, m}=2 \mathrm{~mm}$ & \multicolumn{3}{l}{$\sigma_{\varepsilon, m}=1 \mathrm{~mm}$} \\
& $\operatorname{Pr}(F \mid Z)$ & $\beta$ & $\operatorname{Pr}(F \mid Z)$ & $\beta$ \\
\cline { 2 - 5 }$u_{x, m}=10 \mathrm{~mm}$ & $2.18 \times 10^{-1}$ & 0.78 & $3.31 \times 10^{-1}$ & 0.44 \\
$u_{x, m}=5 \mathrm{~mm}$ & $2.09 \times 10^{-2}$ & 2.04 & $3.59 \times 10^{-2}$ & 1.80 \\
$u_{x, m}=2 \mathrm{~mm}$ & $6.74 \times 10^{-3}$ & 2.47 & $1.84 \times 10^{-3}$ & 2.90 \\
\hline
\end{tabular}

Not surprisingly, for measurements significantly higher than the expected value, the updated failure probability is higher than the prior probability. This difference is more pronounced when the measurement device is more accurate, i.e. when $\sigma_{\varepsilon, m}$ is smaller. For measurements lower than the expected value, the updated failure probability is lower than the prior probability. Again, the difference increases with decreasing value of $\sigma_{\varepsilon, m}$, because this implies a higher information content of the measurement. It is noted that a measurement that corresponds exactly to the expected value of the deformation would lead to a posterior failure probability that is lower than the prior probability, due to a reduction of uncertainty.

The analysis assumes in-plane symmetry. This is a valid assumption in the absence of spatial variability considerations. However, due to the random spatial variability of the soil properties, the problem will not be symmetric about the centre-line and so the assumption of symmetry in this case influences the computed reliability. Moreover, since the analysis is performed in 2D, the correlation length in the out-of-plane direction is assumed to be infinite. The influence of the out-of-plane correlation was investigated in [28] for a 3D slope stability example, where it was shown that the $3 \mathrm{D}$ failure modes have a significant influence on the 
slope reliability for most practical applications. To the knowledge of the authors a similar analysis has not been performed for sheet pile walls and therefore the impact of this assumption on the reliability cannot be assessed.

The number of deterministic FE analyses required by the subset simulation ranges between 1900 and 3700, which includes the evaluation of both integrals in Eq. (13). Considering that the number of random variables of the problem is 432 (the dimension of the reliability problem), the number of required deterministic calculations can be viewed as relatively small. A discussion on the efficiency of reliability methods for high dimensional problems can be found in [29], where the performance of the subset simulation is illustrated for a series of benchmark problems. The higher amount of computations is observed in the case where the assumed measurement differs considerably from the expected value (i.e. the case where $\left.u_{x, m}=10 \mathrm{~mm}\right)$. This is due to the small value of the probability $\operatorname{Pr}\left(Z_{e}\right)$ in Eqs. (12) and (13), resulting in a larger number of levels $M$ in the corresponding run of the subset simulation algorithm.

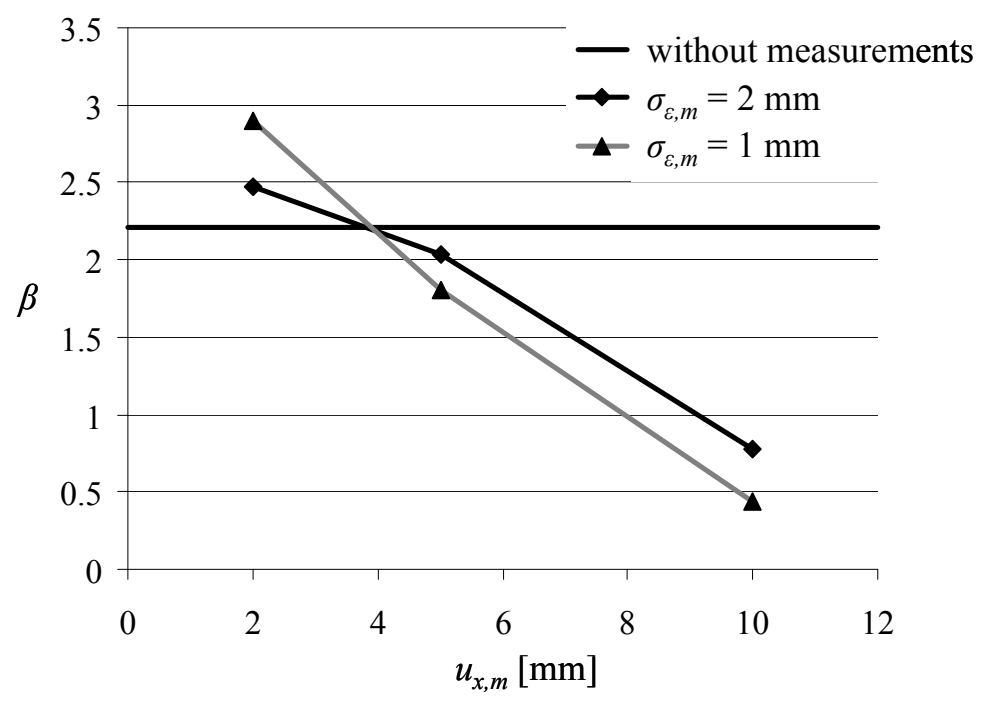

Fig. 6. Reliability index against measured displacement. 
For practical implementation, the reliability can be computed conditional on different hypothetical measurement outcomes, prior to the in-situ measurement. Then a threshold value for the actual measurement may be obtained as a function of the target reliability index $\beta^{T}$ as illustrated in Fig. 7. Assuming that the target reliability is $\beta^{T}=2.5$ and the measurement accuracy is $\sigma_{\varepsilon, m}=1 \mathrm{~mm}$, the threshold value is $3.1 \mathrm{~mm}$. Any measurement larger than this value corresponds to a reliability index less than the acceptable one. This would indicate that the retaining wall would not satisfy the reliability requirements at the final excavation stage and additional measures (e.g. anchors) would be necessary.

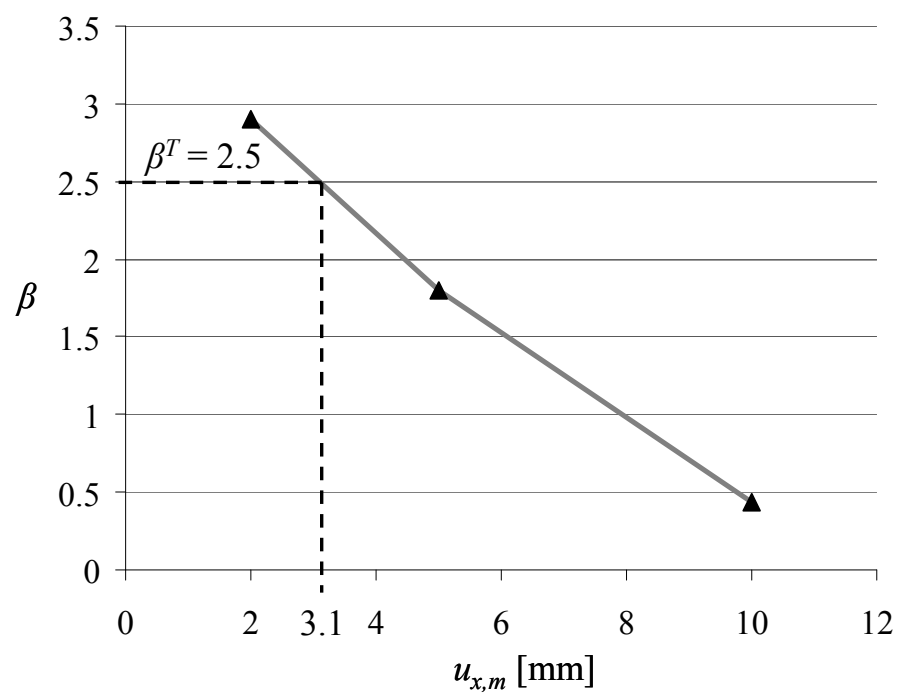

Fig. 7. Evaluation of a threshold measurement value for an accepted reliability index $\beta^{T}$ $=2.5$ and a measurement device with $\sigma_{\varepsilon, m}=1 \mathrm{~mm}$ 


\section{Conclusion}

In this paper, a procedure for the reliability updating of geotechnical sites using measurement information is presented. The applied methodology includes the stochastic discretization of the non-Gaussian random fields, representing the spatial variability of the uncertain material parameters. For the reliability updating, a recently proposed method that tackles Bayesian updating with equality information is applied. This approach is combined with the subset simulation, an adaptive Monte Carlo method that is able to handle efficiently reliability problems with a large number of random variables.

The proposed procedure is applied to a nonlinear stochastic finite element model of an excavation with a sheet pile retaining wall. Different deformation measurement outcomes at an intermediate construction stage are considered. The results show that estimates of the conditional failure probability may be obtained with a feasible computational cost, even when including a large number of random variables for the random field representation of the soil properties. It is also demonstrated how reliability updating computations can be utilized to decide upon the acceptability of a geotechnical design in light of new observations.

\section{Acknowledgments}

This work is partially supported by SOFiSTiK AG. This support is gratefully acknowledged. 


\section{References}

[1] Straub, D. Reliability updating with equality information. Probabilistic Engineering Mechanics, 26(2) (2011), pp. 254-258.

[2] Chowdhury, R. N., Zhang, S., Flentje, P. Reliability updating and geotechnical backanalysis. In: Proc. Skempton Conference on Advances in Geotechnical Engineering, London, March, 2004. London: Thomas Telford Ltd.

[3] Schweckendiek, T., Kanning, W. Updating piping probabilities with survived loads. In: Proc. $7^{\text {th }}$ International Probabilistic Workshop, Delft, November, 2009. Delft: TU Delft.

[4] Ching, J., Hsieh, Y.-H. Updating future reliability of nonlinear systems with low dimensional monitoring data using short-cut simulation. Computers and Structures 87(13-14) (2009), pp. 871-879.

[5] Rackwitz, R. Reviewing probabilistic soils modelling. Computers and Geotechnics 26 (2000), pp. 199-223.

[6] Papaioannou, I, Heidkamp, H, Düster, A, Rank, E, Katz, C. Random field reliability analysis as a means for risk assessment in tunnelling. In: Proc. $2^{\text {nd }}$ International Conference on Computational Methods in Tunnelling EURO:TUN 2009, Bochum, September, 2009. Freiburg: Aedificatio Publishers.

[7] Griffiths, DV, Huang, J, Fenton, GA. Influence of the spatial variability on slope stability using 2-D random fields, J. Geotech. Geoenviron. Engrg., ASCE 135(10) (2009), pp. 1367-1378.

[8] Au, SK, Beck, JL. Estimation of small failure probabilities in high dimensions by subset simulation. Probabilistic Engineering Mechanics 16(4) (2001), pp. 263-277.

[9] Zienkiewicz, OC, Taylor, RL. The Finite Element Method. Fifth edition. Oxford: Butterworth-Heinemann, 2000. 
[10] SOFiSTiK AG, SOFiSTiK analysis programs version 2010. Oberschleißheim: SOFiSTiK AG, 2010.

[11] Grigoriu, M. Crossings of non-Gaussian translation processes. Journal of Engineering Mechanics, ASCE 110(4) (1984), pp. 610-620.

[12] Li, C-C, Der Kiureghian, A. Optimal discretization of random fields. Journal of Engineering Mechanics, ASCE 119(6) (1993), pp. 1136-1154.

[13] Der Kiureghian, A, Liu, P-L. Structural reliability under incomplete probability information. Journal of Engineering Mechanics, ASCE 112(1) (1986), pp. 85-104.

[14] Vanmarcke, E.H. Random Fields: Analysis and Synthesis. Cambridge, MA: MIT Press, 1983.

[15] Sudret, B, Der Kiureghian, A. Stochastic Finite Elements and Reliability: A State-of-theArt Report. University of California, Berkeley, 2000 - Technical Report no UCB/SEMM-2000/08.

[16] Der Kiureghian, A, Ke, J-B. The stochastic finite element method in structural reliability. Probabilistic Engineering Mechanics 3(2) (1988), pp. 83-91.

[17] Ditlevsen, O, Madsen, H. O. Structural Reliability Methods, Chichester, UK: Wiley, 1996.

[18] Zhang, Y, Der Kiureghian, A. Dynamic response sensitivities of inelastic structures. Comput. Methods Appl. Mech. Engrg 108 (1993), pp. 23-36.

[19] Der Kiureghian, A., Zhang, Y. Space variant finite element reliability analysis. Comput. Methods Appl. Mech. Engrg 168 (1999), pp. 173-183.

[20] Katafygiotis, LS, Cheung, SH. Application of spherical subset simulation method and auxiliary domain method on a benchmark reliability study. Structural Safety 29 (2007), pp. 194-207.

[21] Bucher, C. Asymptotic sampling for high-dimensional reliability analysis. Probabilistic Engineering Mechanics 24 (2009), pp. 504-510. 
[22] Metropolis, N., Rosenbluth, A. W., Rosenbluth, M. N., Teller, A. H., Teller, E. Equations of state calculations by fast computing machines. Journal of Chemical Physics 21(6) (1953), pp. 1087-1092.

[23] Hastings, W. K. Monte Carlo sampling methods using Markov chains and their applications. Biometrika 57(1) (1970), pp. 97-109.

[24] Papaioannou, I, Heidkamp, H, Düster, A, Rank, E, Katz, C. Integration of reliability methods into a commercial finite element software package. In: Proc. $10^{\text {th }}$ International Conference on Structural Safety and Reliability ICOSSAR 2009, Osaka, September, 2009. London: Taylor \& Francis Group.

[25] Tschebotarioff, GP. Soil Mechanics, Foundations and Earth Structures. New York: McGraw-Hill, 1951.

[26] Jaky, J. The coefficient of earth pressure at rest. In Hungarian. J. Soc. Hung. Eng. Arch. (1948), pp. 355-358.

[27] Matsui, T, San, KC. Finite element slope stability analysis by shear strength reduction technique. Soils Found., 32(1) (1992), pp. 59-70.

[28] Hicks, MA, Spencer, WA. Influence of heterogeneity on the reliability and failure of a long 3D slope. Computers and Geotechnics 37 (2010), pp. 948-955.

[29] Schuëller, GI, Pradlwarter, HJ. Benchmark study on reliability estimation in higher dimensions of structural systems - An overview. Structural Safety 29 (2007), pp. 167182. 